P607

DETECTION OF MYCOPLASMA GENITALIUM MACROLIDE RESISTANCE USING THE OPEN CHANNEL OF THE PANTHER FUSION ${ }^{\circledR}$ SYSTEM

${ }^{1}$ Robert Kulis-Horn, ${ }^{2}$ Klaus Jansen, ${ }^{3} J$ orgen Jensen, ${ }^{4}$ Carsten Tiemann*. ${ }^{1}$ Krone Laboratory, Molecular Diagnostics, Bad Salzuflen, Germany; ${ }^{2}$ Robert Koch Institute, Infectious Disease Epidemiology, Berlin, Germany; ${ }^{3}$ Statens Serum Institut, DK, Denmark; ${ }^{4}$ Krone Laboratory/ LABCON-OWL, Molecular Diagnostics, Bad Salzuflen, Germany

\subsection{6/sextrans-2019-sti.675}

Background Current epidemiological studies demonstrate a high prevalence of Mycoplasma genitalium (MG) infections in high-risk groups, especially MSM. Owing to the widespread macrolide resistance the European Guideline on MG infections recommend complementing the molecular detection of $\mathrm{MG}$ with an assay capable of detecting macrolide resistance-associated mutations. Macrolide resistance is caused by a single nucleotide polymorphism (SNP) in region $\mathrm{V}$ of the $23 \mathrm{~S}$ rRNA gene. Two nucleotide positions in the $23 \mathrm{~S}$ rRNA gene have been associated with azithromycin resistance: A2058 and A2059.

Methods The Aptima M. genitaliumAssay ${ }^{\circledR}$ was used for the initial detection on the Panther ${ }^{\circledR}$ system. A hydrolysis probe real-time PCR on theOpen Channelof the PantherFusion ${ }^{\circledR}$ system for automated reflex testing after MG positive results was established and validated. The Open Channelof the instrument allows the use of custom primers and probes on the PantherFusion $^{\circledR}$ system. Minor groove binder (MGB) hydrolysis probes were used for accurate and reliable SNP discrimination at position 2058/2059. The workflow enables an automated analysis process including DNA extraction, PCR setup, and results interpretation. Using the open channel 300 samples could be genotyped within an 8 h working day.

Results $60 \mathrm{MG}$ positive clinical samples were tested. The laboratory developed test (LDT) was able to detect the wild type variant in 20 samples and the A2058G/A2059G mutations in 22 samples. All results were confirmed by amplicon sequencing and commercially available test system. 18 (30\%) samples did not show any typing result either using different LDT or commercial test system.

Conclusion MG positive samples can be typed for macrolide resistance using our LDT on the same platform during one run. The combination of a MG high-throughput test followed by macrolide resistance testing improves the efficiency of large-scale epidemiological resistance surveillance. However, highly sensitive TMA assay might result in a significant number of non-typable samples. Further studies are needed to improve the sensitivity and explanatorypower of MG resistance testing.

Disclosure No significant relationships.

\section{P608 SIMPLEPROBE PCR ASSAY FOR DETECTION OF MUTATIONS ASSOCIATED WITH MACROLIDE RESISTANCE IN MYCOPLASMA GENITALIUM SAMPLES}

Marianne Gossé, Hilde Lysvand, Brita Pukstad, Svein Arne Nordbø. Norwegian University of Science and Technology, Department of Clinical and Molecular Medicine, Trondheim, Norway

10.1136/sextrans-2019-sti.676

Background Macrolide-resistant strains of Mycoplasma genitalium are an increasing problem throughout the world, and the implementation of a rapid and sensitive assay for mutation detection to guide treatment is needed. Macrolide-resistant strains have been shown to contain base substitutions in positions 2058 and 2059 (Escherichia coli numbering) in region V of the $23 \mathrm{~S}$ rRNA gene. In this study, we present a SimpleProbe PCR followed by melting curve analysis to differentiate between macrolide-resistant mutants and wild types.

Methods The assay was performed on 159 Mycoplasma genitalium-positive samples, and the results were compared with DNA sequencing. We also looked at the prevalence of macrolide-resistant strains in a Norwegian population.

Results Of 139 samples characterized successfully by sequencing, $54(39 \%)$ were wild types and 85 (61\%) were mutants, consisting of 59 (42\%) A2059G, 24 (17\%) A2058G, 1 (1\%) $\mathrm{A} 2058 \mathrm{~T}$, and 1 (1\%) A2059C mutation. The melting curve analysis correctly differentiated between wild-type and mutant strains in all cases, but it could not identify the different mutant types.

Conclusion The SimpleProbe PCR proved to be a simple, rapid, and reliable method for the detection of macrolideresistant isolates of Mycoplasma genitalium in a clinical setting.

Disclosure No significant relationships.

\section{P609 CLINICAL EVALUATION OF THREE COMMERCIAL PCR ASSAYS FOR THE DETECTION OF MYCOPLASMA GENITALIUM AND MACROLIDE RESISTANCE}

${ }^{1}$ Chloe Le Roy, ${ }^{1}$ Cécile Bébéar, ${ }^{2}$ Sabine Pereyre. ${ }^{1}$ University of Bordeaux, Bordeaux, France; ${ }^{2}$ University of Bordeaux, USC-EA 3671 Mycoplasmal and Chlamydial Infections in Humans, Bordeaux, France

\subsection{6/sextrans-2019-sti.677}

Background Because macrolide resistance is increasing worldwide in Mycoplasma genitalium (MG), it is recommended to detect macrolide resistance-associated mutations in MG-positive specimens. Some new commercial kits detect macrolide resistance at the same time as MG detection. The aim the study was to prospectively evaluate the clinical performance of three commercial kits for the detection of $\mathrm{MG}$ and macrolide resistance.

Methods Two hundred MG-positive urogenital specimens detected using an in-house real-time PCR are prospectively collected at the Bacteriology Department of Bordeaux University Hospital (France). After DNA extraction of the specimens using the MagNa Pure 96 (Roche), the specimens are submitted to four assays: the SpeeDx ResistancePlus MG assay, the Diagenode S-DiaMGRES assay, the PathoFinder Real accurate TVMG $^{\text {RES }}$ and the in-house FRET qPCR assay and $23 \mathrm{~S}$ rRNA sequencing used as reference. The internal controls of each kits were added in the specimen before extraction and the absence of amplification inhibition associated with the addition of the three internal controls was previously checked.

Results To date, 114 MG-positive specimens have been analyzed. The clinical sensitivity for MG detection was similar for the three commercial kits, ranging between 95.5 and $98.2 \%$. To compare the macrolide resistance detection performance, 90 specimens that have been found MG-positive using the three kits and that could be amplified using the inhouse FRET qPCR assay were retained. Therefore, compared 
to the reference assay, clinical sensitivity of the three commercial kits for the detection of resistance was ranging between $89.1 \%$ and $100 \%$ and clinical specificity was ranging between 93.2 and $97.7 \%$, with no statistical significant difference. Testing additional urogenital specimens will allow to specify these percentages.

Conclusion The launch of three sensitive and specific commercial kits for the detection of MG and macrolide resistance will be useful to guide the choice of therapy.

Disclosure No significant relationships.

\section{P610 MYCOPLASMA GENITALIUM IN CLIENTS VISITING TWO DUTCH STI CLINICS: VERY HIGH PREVALENCE AND RESISTANCE TO AZITHROMYCIN}

\begin{abstract}
${ }^{1}$ Sylvia Bruisten*, ${ }^{2}$ David Hetem, ${ }^{3}$ Jean-Marie Brand, ${ }^{4}$ Martijn Van Rooijen, ${ }^{4}$ Paul Oostvogel, ${ }^{4}$ Clarissa Vergunst, ${ }^{2}$ Roel Nijhuis, ${ }^{3}$ Sophie Kuizenga-Wessel, ${ }^{1}$ Alje Van Dam. ${ }^{1}$ Public Health Service Amsterdam, Amsterdam University Medical Center (UMC), Infectious Diseases, Infection and Immunity (AI and II), Amsterdam, Netherlands; ${ }^{2} \mathrm{HMC}$ Westeinde, Medical Microbiology, Den Haag, Netherlands; ${ }^{3}$ Public Health Service Haaglanden, Infectious Diseases, Den Haag, Netherlands; ${ }^{4}$ Public Health Service of Amsterdam, Infectious Diseases, Amsterdam, Netherlands
\end{abstract}

\subsection{6/sextrans-2019-sti.678}

Background Associations between Mycoplasma genitalium $(\mathrm{MG})$ and male urethritis and pelvic inflammatory disease are reported frequently. The recent prevalence of MG among clients of Dutch STI clinics is unknown. Treatment is hampered by resistance to azithromycin.

Methods Urogenital samples from 939 men who have sex with women (MSW), 1025 men who have sex with men (MSM) and 1273 women were included at the STI clinics of Amsterdam and the Hague. Also anal samples from all MSM and from 984 women were included. TMA assays (Aptima, Hologic) were used to detect MG, Chlamydia trachomatis (CT) and Neisseria gonorrhoeae (NG). Azithromycin resistance was tested using a new qPCR with LNA probes and confirmed by 23SrRNA gene sequencing.

Results The overall MG prevalence among STI clients was $19.9 \%$ in MSM, $8.4 \%$ in MSW and $12.5 \%$ in women. Prevalence for all risk groups was higher in STI clients in Amsterdam than in the Hague. MG-positivity was detected in $8.4 \%$ urines of MSW and $6.9 \%$ of MSM. In women, MG was found in $11.0 \%$ of vaginal samples. Of the anal samples of MSM $15.4 \%$ and of women $10.0 \%$ were MG positive. Among MG-positive MSM $67.5 \%$ and among MG-positive women $16.1 \%$ were $M G$-positive in the anal sample only. $M G$ and NG were associated in men $(\mathrm{p}<0.001)$, but not MG and CT. Detected azithromycin resistance was very high: 34/50 (68\%) of urines, 48/81 (59\%) of anal samples and $37 / 75$ (49\%) of vaginal samples.

Conclusion MG is highly prevalent in Dutch STI clinic clients and resistance frequency to azithromycin is very high. In MSM, isolated anal MG is common, but frequently missed if left untested. Anal MG in women is also common, but testing vaginal MG detects most cases. In men, NG and MG infections are associated. Information on clinical relevance of $\mathrm{MG}$ and azithromycin resistance is urgently needed.

Disclosure No significant relationships.
P611 HIGH SEROPREVALENCE OF MYCOPLASMA GENITALIUM IN THE GENERAL ADULT POPULATION OF GERMANY

${ }^{1}$ Felix Reichert*, ${ }^{2}$ Tim Waterboer, ${ }^{3}$ Christina Poethko-Müller, ${ }^{1}$ Klaus Jansen. ${ }^{1}$ Robert Koch Institute, Infectious Disease Epidemiology, Berlin, Germany; ${ }^{2}$ German Cancer Research Center (DKFZ), Infections and Cancer Epidemiology, Heidelberg, Germany; ${ }^{3}$ Robert Koch Institute, Epidemiology and Health Reporting, Berlin, Germany

\subsection{6/sextrans-2019-sti.679}

Background Mycoplasma genitalium (MG) is one of the most common bacterial sexually transmitted infections (STI). Nevertheless, knowledge about the immune response is scarce and seroprevalence has only been assessed in selected populations. In HIV-negative blood donors seroprevalence was 5.5\%, in patients with pelvic inflammatory disease 17\%. We estimated MG seroprevalence in the general adult population in Germany.

Methods We retrospectively analysed sera of the populationbased German National Health Interview and Examination Survey 1998 for MG antibodies using a pre-validated, multiplex, fluorescent bead-based assay. To avoid cross-reactivity, two unconserved regions of adhesion proteins, $\mathrm{MgPaN}$ and $\mathrm{rMgPa}$ were chosen as antigens. The thresholds for seropositivity were set at 1000 median fluorescence intensity units by visual inspection of inflection points. Overall seropositivity for MG was defined as concomitant seropositivity for both antigens. To assure representativeness, survey weights were applied for calculation of prevalence and 95\% confidence intervals (CI). Design-based F was computed as test of independence.

Results Sera of 6038 participants were analysed. Participants were aged 17-79 years (y) (median 44y), 51\% were female. Overall weighted seroprevalence was $6.5 \%$ (95\%CI: $5.7-$ $7.3 \%)$, and by age-group $17-19 y \quad 0.3 \%$ (0.1-1.5\%), 20-24y $1.9 \% \quad(0.9-3.8 \%), \quad 25-29 y \quad 6.7 \% \quad(4.6-9.8 \%), 30-39 y \quad 9.1 \%$ (7.4-11.1\%), 40-49y 10.8\% (8.9-13.0\%), 50-59y 4.5\% (3.4$5.9 \%), \quad 60-69 y \quad 3.3 \% \quad(2.2-5.1 \%)$, and $70-79 y \quad 4.6 \% \quad(2.9-$ $7.4 \%)$. In the age-group $20-29 y$, women had a higher seroprevalence $(7.5 \%, 95 \% \mathrm{CI}: 5.0-11.1 \%)$ than men $(2.1 \%, 95 \% \mathrm{CI}$ : $1.1-4.0 \% ; \mathrm{p}=0.001)$.

Conclusion Compared to other studies, our results for $\mathrm{MG}$ seroprevalence in the general adult population in Germany are plausible.

The earlier increase in women is similar to the pattern in other STI such as Chlamydia trachomatis infection. Seroprevalence dropped in participants aged 50 or more but remained stable at a low level until old age, indicating long antibody persistence or continuous sexual interactions in parts of the population. However, longitudinal studies are necessary to clarify immunological processes.

Disclosure No significant relationships.

\section{P612 ANTIMICROBIAL SUSCEPTIBILITY DATA OF MYCOPLASMA GENITALIUM STRAINS ISOLATED IN JAPAN}

${ }^{1}$ Ryoichi Hamasuna, ${ }^{2}$ Masahiro Matsumoto, ${ }^{2}$ Naohiro Fujimoto, ${ }^{2}$ Tetsuro Matsumoto. ${ }^{1}$ ShinKokura Hospital, Urology, Kitakyushu, Japan; ${ }^{2}$ University of Occupational and Environmental Health, Urology, Kitakyushu, Japan

\subsection{6/sextrans-2019-sti.680}

Background The initial culturing of Mycoplasma genitalium from clinical specimens is still difficult and the antimicrobial susceptibility data of $M$. genitalium strains have not been 